\title{
L'ANENA un organisme au service de la sécurité « neige et avalanches »
}

\author{
L. de Crecy \\ Membre fondateur et Vice-Président de l'ANENA (1971-1980) \\ F. Sivardière \\ Directeur de l'ANENA
}

En guise d'introduction aux exposés qui vont suivre ce matin concernant la neige et l'avalanche, les organisateurs ont, avec raison, voulu que vous soit d'abord présentée l'ANENA, cette structure originale qui rassemble entre autres, et sans pourtant rien leur imposer, les différents organismes représentés par les orateurs que vous allez entendre.

Permettez-nous d'abord une courte rétrospective historique où la Société Hydrotechnique de France, qui nous réunit aujourd'hui, apparaît en quelque sorte, comme une ancêtre de l'ANENA.

\section{D LES PROBLÈMES D'AVALANCHES AVANT 1971}

\subsection{La section de glaciologie de la SHF et le CEDONIGLA}

Evoquant l'an dernier la naissance de la section de glaciologie de la SHF en 1948, l'un de nous rappelait qu'elle était née de la volonté de faire se retrouver dans une enceinte neutre d'une part des forestiers de montagne déjà très motivés par la neige et la lutte contre l'avalanche et, d'autre part, les jeunes équipes d'hydro-électriciens qui investissaient à leur tour la montagne pour exploiter la neige source d'énergie.

De fait, les réunions annuelles et les «tournées glaciologiques " très appréciées de notre section devaient devenir, 23 ans durant, les seules occasions de rencontre offertes à tous ceux qui s'intéressaient à la neige à un titre quelconque en France.

Parmi eux, dès 1955, figuraient les membres participants à ce curieux organisme grenoblois baptisé CEDONIGLA (Centre d'Etudes et de Documentation NIvoGLAciologiques) qui, sans avoir d'existence légale, rassemblant déjà forestiers, météorologistes et hydroélectriciens autour de la neige et de l'avalanche, publiait un bulletin et gérait au $\mathrm{Col}$ de Porte le premier laboratoire français pour l'étude de la neige.

\subsection{L'avalanche de Val d'Isère et la Commission d'en-} quête

Lorsque, le 10 février 1970, une avalanche monstrueuse tua les 39 pensionnaires de l'UCPA dans leur chalet à l'heure du petit-déjeuner, l'émotion dans le public fut considérable.
Le Gouvernement nomma une Commission d'enquête. Celle-ci constata bien vite que bien des compétences éparses existaient en France dans le domaine de l'avalanche mais que, sauf dans le cas du CEDONIGLA, leur mauvaise coordination nuisait à leur efficacité.

Désireuse d'y remédier, elle souhaita pouvoir donner à ses conclusions une portée nationale dépassant le cadre de l'affaire de Val d'Isère; elle demanda et obtint de voir élargir sa mission à l'échelle nationale en se transformant en «Commission interministérielle d'études sur la Sécurité en montagne ».

Dans ce nouveau cadre, sa première tâche fut le recensement de tout ce qui se faisait en France dans le domaine de l'avalanche ; à très peu près, il suffisait, pour y parvenir de reprendre la collection des 23 ans de communications à notre section de glaciologie de la SHF.

La Commission va ensuite entendre les responsables de ces différentes équipes, enregistrer leurs préoccupations très diverses et constater leurs divergences sur la lutte contre l'avalanche en France. La suite de la mission de la Commission comportait une visite à l'étranger, en Autriche et en Suisse, pays qui possédaient, à l'époque, une avance considérable dans ce domaine.

La Commission visita, notamment en Suisse, au Weissfluhjoch près de Davos, le fameux Institut Fédéral pour l'Etude de la Neige et des Avalanches, qui lui parut le modèle de ce qu'elle devait chercher à implanter en France : un organisme unique et centralisé où collaborent des physiciens, des hydrodynamiciens, des météorologistes, des forestiers, qui réalise des prévisions du risque dans le temps et dans l'espace, qui élabore et met en œuvre les techniques de protection active et passive, de prévention et de secours ; qui est appelé systématiquement à donner et à imposer - son avis lors des implantations nouvelles d'équipements en montagne, routes, bâtiments ou pistes.

1.3 L'ANENA, un pis-aller provisoire en attendant l'Institut de la neige?

De retour en France, la Commission, éblouie, souhaitait donc vivement jeter les bases du futur «Institut de la Neige " français.

Mais il lui fallait obtenir de chaque organisme qu'il abandonnât sur-le-champ, entre les mains de l'institut à créer, sa documentation, ses sites expérimentaux, ses la- 
boratoires et surtout ses chercheurs et spécialistes expérimentés. Il fallait aussi, du jour au lendemain, trouver les crédits nécessaires à la création en altitude d'importants bâtiments et persuader des chercheurs compétents de venir s'y installer.

Très vite, la Commission dut reconnaître que la prétention de voir surgir rapidement « I'Institut de la neige " français était illusoire.

C'est faute de mieux et à titre provisoire que la Commission se résigna à proposer seulement la création d'une simple association selon la loi de 1901 qui devait être l'ANENA. Le rapport déposé en août fut adopté par le Gouvernement le 21 octobre 1970. Il traçait les grandes lignes du programme de recherches et actions concrètes qui devait être encouragé par l'association, en attendant d'être conduit par le futur Institut.

\subsection{Un an pour constituer l'ANENA !}

Rassembler en vue d'une coopération efficace des organismes jusqu'ici indépendants, voire concurrents n'était pas chose aisée, d'autant que l'ambition était de leur associer le plus possible «d'usagers» de la montagne, communes, randonneurs, skieurs, stations de sports d'hiver.

Beaucoup, notamment à Paris, pensaient qu'un tel rassemblement était parfaitement irréalisable.

Mais nous avions à Grenoble, quelques atouts :

- la concentration dans la région d'un grand nombre d'organismes concernés ;

- la plupart d'entre eux s'étaient rencontrés dans l'une ou l'autre des réunions de la section de glaciologie de la SHF ; - les plus importants laboratoires de recherche collaboraient déjà sur le terrain notamment au CEDONIGLA ; - la bienveillance et le dévouement du Professeur Néel, qui, non spécialiste de la neige, avait pourtant compris l'importance de l'enjeu et accepté de mettre à notre service son immense prestige en devenant notre premier Président. Le doigté et l'autorité avec lesquels il sut à la fois ménager les susceptibilités et imposer les solutions les plus raisonnables fut un des facteurs essentiels de la réussite: le 11 octobre 1971 était créée l'ANENA (Association Nationale pour l'Etude de la Neige et des Avalanches).

Il faut aussi souligner le rôle très important joué par Jean Franco (célèbre alpiniste, créateur de l'ENSA, Inspecteur de la Jeunesse et des Sports) qui a rédigé les statuts de l'ANENA à travers lesquels il a permis à tous les acteurs impliqués par le problème des avalanches d'être représentés. Il fut le premier Directeur de l'ANENA, mais devait malheureusement décéder deux mois après sa nomination. Toutefois, son enthousiasme, sa foi passionnée dans la tâche qui lui était impartie, ont ouvert la voie à ses successeurs et donné l'impulsion qui conduit au succès.

\section{II — LES PREMIERS PAS DE L'ANENA}

\subsection{La composition du premier Conseil d'administration}

\section{Les scientifiques}

Chargée de faire progresser les connaissances et leur diffusion, l'association devait d'abord être l'affaire des scientifiques. L'adhésion des principaux laboratoires concernés ne posa pas de problème majeur: l'appui du Professeur Néel, l'acquis du CEDONIGLA, les liens tissés à la section de glaciologie de la SHF et, peut-être, la perspective de quelque attribution nouvelle de crédits supplémentaires la facilitèrent.

\section{Les administrations}

Un très grand nombre de ministères s'estimaient concernés: L'Intérieur, par la Sécurité civile et les CRS, les Armées par la Gendarmerie et les Troupes de montagne, l'Agriculture et ses forestiers, l'Equipement, l'Aménagement du Territoire, l'Aviation civile dont dépendait la Météorologie Nationale, le Commissariat au Tourisme, l'Environnement, l'Education Nationale car il y avait des techniciens à former... Il y aurait eu plutôt pléthore de candidatures de la part des ministères sans la perspective d'avoir à subventionner.

\section{Les usagers de la montagne}

Le nouvel organisme avait à diffuser les connaissances dans le public et chez les responsables locaux pour induire des comportements adaptés aux risques ; leur participation était essentielle mais les réticences furent sensibles. La première réunion, le 11 octobre 1971 , ne rassemblait que deux députés de montagne, un représentant du Comité des Stations de sports d'hiver, un représentant de la Fédération Française de la Montagne et un entrepreneur pyrénéen spécialisé dans le montage d'ouvrages paravalanches.

\subsection{Etudier l'avalanche sans la nommer?}

Encore fallut-il faire admettre que le nom de l'association pût comporter le mot avalanche! On voulait bien que l'avalanche fût un objet d'étude prioritaire, mais, pour certains responsables des stations de ski de l'époque, il ne fallait surtout pas le proclamer publiquement de peur de terrifier les clients ! L'autorité du Professeur Néel ne fut pas de trop pour faire prévaloir la solution raisonnable!

Cependant, les stations les plus conscientes comprirent vite que leur intérêt était d'adhérer et de participer aux travaux de l'ANENA.

\subsection{L'ANENA, une carte de visite efficace !}

Avant la création de l'ANENA, les techniciens de l'Etat ayant quelques compétences en matière de neige et d'avalanche avaient parfois tenté de venir proposer, gratuitement, leurs services aux stations qui leur paraissaient avoir des problèmes dans ce domaine.

Mais « l'Administration " faisait si peur que, de manière quasi systématique, ils se voyaient plus ou moins poliment éconduits... pour apprendre, à leur retour que leur visite avait eu au moins le mérite d'inquiéter, puisque leurs collègues suisses avaient presque aussitôt été appelés en consultation - à grands frais - sur les mêmes problèmes.

Mais, dès la création de l'ANENA tout fut changé : cotisant à une association spécialisée, les stations eurent tout naturellement recours à ses services et accueillirent avec gratitude sous la casquette de l'ANENA les mêmes hommes qu'ils auraient évincés comme représentants de l'Etat.

Au fait, un « Institut de la neige » officiel n'aurait-il pas fait peur aux usagers plus qu'une association? 


\section{LES MULTIPLES RÔLES DE L'ANENA}

Réunissant des scientifiques, des administrateurs et des usagers, l'ANENA ne pouvait ni ne voulait se substituer à ses membres, même si ceux-ci gagnaient en efficacité à se présenter sous ses couleurs.

\subsection{L'accroissement des moyens scientifiques}

Au plan de la recherche, fondamentale et appliquée, le CNRS et l'Université ont continué à financer les études du laboratoire grenoblois de glaciologie, la Météorologie à affiner ses méthodes de prévisions, les services de l'Agriculture et de l'Equipement à expérimenter les ouvrages de protection et les méthodes de déclenchement artificiel comme à parfaire les techniques de zonages de risques.

Mais, au sein de l'ANENA, ces actions et ces programmes étaient présentés, expliqués, discutés devant l'ensemble des usagers.

Ceux-ci, en saisissant mieux l'intérêt et les retombées prévisibles, exerçaient une pression " politique " sensible sur les décideurs aux échelons ministériel et gouvernemental. D'où un accroissement substantiel des moyens matériels et humains mis à la disposition des chercheurs et des progrès rapides dans l'élaboration des nouvelles techniques.

\subsection{La mise en æuvre des techniques : l'ANENA groupe de pression vis-à-vis de l'Administration !}

Mais le progrès des techniques ne suffit pas toujours. Encore fallait-il que leur mise en œuvre fût possible du point de vue légal et réglementaire !

Pour prendre un exemple, on n'imagine pas le nombre de réunions que l'ANENA dut organiser à Paris auprès des cabinets ministériels pour que des techniques aussi courantes à l'étranger que les déclenchements préventifs d'avalanches devinssent possibles chez nous.

En effet, il était a priori tout à fait inimaginable, pour un administrateur français normal, que des personnes privées (responsables de stations) puissent être autorisées à détenir des dizaines de kilos d'explosifs, à décider ellesmêmes, seules et sur-le-champ, pendant ou juste après une chute de neige de les faires amorcer par des personnels bien formés, certes, par l'ANENA, mais parfois sans diplômes préalables scolaires ni universitaires, de les acheminer, pré-amorcées vers des sites éloignés, d'accès presque toujours dangereux, par les moyens les plus variés, skieurs, câbles, lanceurs de projectiles ou hélicoptères, afin de déclencher volontairement des avalanches au-dessus de pistes ou de routes fréquentées par les touristes.

Le luxe des précautions, apparemment «tatillonnes", qu'imposent les «PIDA » (Plan d'Intervention pour le Déclenchement d'Avalanches) aux stations qui utilisent ces techniques reflète l'inquiétude très vive des autorités contraintes de les réglementer. Mais il témoigne aussi, et par là même, du " poids " de l'ANENA qui, seule, était capable d'ajouter à des arguments techniques convaincants assez d'impact médiatique et donc d'appuis politiques pour emporter la décision là où une administration isolée aurait échoué à coup sûr.

\subsection{L'ANENA et la formation de spécialistes}

La demande de connaissance et de formation, dans un domaine nouveau dont les stations découvraient l'importance économique et surtout l'impact psychologique sur le public, fut considérable.
Avec l'aide des rares spécialistes de la Météo, de la Division Nivologie du CEMAGREF et du Laboratoire d'Applications Spéciales de la Physique du CENG, l'ANENA organisa très vite des stages de formation de différents niveaux pour diffuser des connaissances de base et des techniques diverses: nivologie et sondages stratigraphiques dans le manteau neigeux pour la prévision, divers modes de déclenchements d'avalanches avec le fameux et très demandé brevet de pisteur-artificier, procédés de recherches de victimes par sondages, détecteursradio, formation de maîtres-chiens d'avalanche, secourisme en avalanche, etc.

Le succès de ces stages fut considérable et permit à notre pays de combler, en quelques années un retard considérable dans ce domaine, au point que l'ANENA fut bientôt sollicitée pour organiser les mêmes types de stages chez certains de nos voisins.

La collection des 68 numéros parus de la revue de l'ANENA, "Neige et Avalanches " témoigne du souci permanent de formation des cadres et de mise à jour de leurs connaissances qui anime l'association. L'accueil fait par l'étranger à cette publication est un garant de sa valeur.

\subsection{L'ANENA et le public}

Faire avancer les connaissances sur la neige et diffuser les techniques auprès des responsables était indispensable mais, en matière d'avalanches, c'était insuffisant puisque, dans $80 \%$ des accidents graves, le comportement personnel des victimes peut être mis en cause, qu'il s'agisse de skieurs de randonnée ou de hors-pistes.

Après la prestigieuse présidence d'un prix Nobel, le Professeur Néel, après celle d'un grand administrateur, le Préfet Saunier, ancien Président de la Commission dite " de Val d'Isère ", l'ANENA marqua son souci d'être au service des randonneurs en se donnant comme Président le Professeur Traynard qui fut l'un des grands dirigeants du Club Alpin Français et dont les célèbres descriptions d'itinéraires de ski de montagne sont, depuis plusieurs décennies, la « bible " des randonneurs alpins.

Sous sa présidence, l'ANENA a poussé à la création d'un réseau toujours plus dense de répondeurs téléphoniques nivo-météorologiques, elle a fait réaliser et imposer un type d'appareil radio de recherche de victimes qui doit désormais être porté par tout randonneur (I'ARVA, Appareil de Recherche de Victimes d'Avalanches).

L'ANENA a multiplié, dans la mesure de ses moyens, les dépliants et brochures de vulgarisation. Pour le ski de station, le public à informer est encore beaucoup plus large mais le message est plus simple, puisqu'il ne lui est demandé que de connaître (et de respecter !) les consignes données par la station notamment sur les fermetures des pistes et autres «domaines skiables ». Même si l'expérience prouve, hélas, que le respect de ces consignes n'est pas toujours facile à obtenir, il reste que l'ANENA a . beaucoup fait et continue d'œuvrer au sein d'organismes internationaux (C.I.S.A./I.K.A.R., Commission Internationale de Sauvetage Alpin, FIPS, Fédération Internationale des Patrouilles à Ski) pour uniformiser et codifier ces messages (panneaux, drapeaux, logos), afin qu'ils soient reçus et compris par tous les publics. 


\subsection{L'ANENA, espace de dialogue et de négociation}

La véritable vocation de l'association est bien, finalement d'être un lieu de rencontre, une occasion de dialogue, un terrain de négociations entre des partenaires ayant le même centre d'intérêt mais des intérêts propres parfois divergents ! Négociations entre les scientifiques et les administrations, entre les administrations et les autorités locales, maires de stations et responsables de pistes ou de remontées, rencontres entre les théoriciens et les praticiens, dialogue, enfin et surtout, entre ceux qui sont réputés «savoir» et les différents publics avides de comprendre et de conjurer ce qu'a encore d'irrationnel pour beaucoup de skieurs le mystérieux risque d'avalanche.

A l'évidence, si le chemin parcouru est immense, il reste encore énormément à faire ! Mais il est temps de dresser un rapide tableau de ce qui est actuellement entrepris sous l'égide de l'ANENA.

\section{IV — L'ANENA EN 1995}

\subsection{Un bref retour en arrière}

Chacun des présidents successifs a donné à l'Association une orientation politique déterminée par les priorités du moment. Avec le recul, ces différentes politiques apparaissent très complémentaires. La nouvelle association fut d'abord une affaire de spécialistes. L'accent fut mis tout naturellement sur la recherche et la progression des connaissances. Au sein du premier Conseil d'Administration présidé par le Professeur Louis Néel (Directeur du CENG et prix Nobel de physique), les représentants des usagers n'occupaient que quelques strapontins.

Avec le Président Saunier (1976-1978) vint l'ère du dialogue et des négociations avec les administrations qu'il fallut convaincre de la nécessité de réformer certaines réglementations (par exemple en matière de réglementation sur les explosifs). 1976 fut aussi l'année de la reconnaissance d'utilité publique de l'ANENA, la confortant ainsi dans le rôle qui lui avait été attribué par le Gouvernement en 1970.

Philippe Traynard (1978-1983), Directeur de l'Institut Polytechnique de Grenoble, n'oublia pas qu'il était aussi skieur de montagne et il intensifia les actions d'informations en direction des usagers « indépendants » de la montagne hivernale, suivi dans cette voie par son successeur Pierre Corbet (1983-1988).

Enfin la présidence de l'ANENA échut pour la première fois à un élu, Jean-Guy Cupillard, maire de l'Alpe d'Huez, qui en assure toujours la responsabilité. Ce changement dans la continuité a été bénéfique ; sans doute est-il l'heureux indice que les élus de la montagne ne voient plus dans l'ANENA un organisme donnant volontiers dans le «catastrophisme ", mais un outil au service de l'ensemble des montagnards.

\subsection{Et maintenant?}

L'ANENA remplit aujourd'hui quatre fonctions essentielles, qu'elle n'a, en fait, jamais cessé d'assurer. De par sa nature même, elle est un espace de concertation. Elle anime, coordonne et gère des recherches. Elle forme et informe.

\subsubsection{Un espace de concertation}

L'ANENA n'est pas une administration. Structure légère, souple, elle tire son originalité et sa force de la co-existence en son sein de toute une série d'acteurs concernés à un titre ou à un autre par la neige et les avalanches.

Au sein du Conseil d'Administration et des différentes Commissions mises en place cohabitent les organismes de recherche (Centre d'Etudes de la Neige de Météo France et Division Nivologie du CEMAGREF principalement, mais aussi Laboratoire d'Applications Spéciales de la Physique du CENG, Centre de Droit du Tourisme et de la Montagne), des Elus (des stations de sports d'hiver, de communes de montagne, des départements), des professionnels de la montagne (pisteurs-secouristes, gendarmes, CRS, directeurs de pistes, de remontées mécaniques, des guides, des moniteurs de ski), des industriels et bien entendu des usagers, skieurs ou randonneurs.

Réunions de travail, tables rondes et autres symposiums permettent d'établir des contacts à tous les niveaux, notamment avec les spécialistes étrangers poursuivant des objectifs similaires.

Ont ainsi récemment pu voir le jour le dépliant sur les " 10 règles de conduite du skieur sur piste ", l'échelle européenne du risque d'avalanches, le sac avalanche (dans le cadre de la normalisation de la signalisation sur les avalanches). Sont actuellement en cours ou prévus, des travaux sur la normalisation de la signalétique des pistes de ski, la rédaction d'un dictionnaire des termes « neige et avalanches ».

En ce qui concerne les colloques et autres congrès, l'ANENA a pendant longtemps organisé des journées annuelles où les participants se réunissaient autour d'un thème. Depuis le début des années 1990, l'Association s'oriente vers la formule «symposium international de 5 jours » tous les quatre ans. En 1991, il réunissait 185 participants de 11 nationalités différentes pour 35 exposés, des démonstrations de matériels et de logiciels. Le succès de cette formule a incité l'ANENA à réorganiser un tel symposium en 1995 à Chamonix. Ainsi, pendant 5 jours, les spécialistes internationaux de la neige et des avalanches vont se rencontrer, faire le point sur l'état des connaissances et de la recherche. C'est aussi l'occasion pour des personnes qui ne rencontrent pas habituellement de le faire, par exemple de permettre aux chercheurs de faire part de leurs travaux aux praticiens, et à ces derniers d'orienter les prochaines recherches afin qu'elles correspondent à leurs "problèmes de terrain ».

\subsubsection{Un partenaire pour la recherche}

L'ANENA n'est pas un laboratoire de recherche ayant ses propres moyens. Le rôle que l'ANENA joue dans ce domaine est celui d'un catalyseur. Elle sert de support administratif et gère des programmes de recherche associant plusieurs laboratoires, notamment le CEN/Météo France et le CEMAGREF, Division Nivologie.

Toutefois par ces contacts avec les « hommes de terrain ", elle n'hésite pas à faire remonter dans les laboratoires des études à l'écart de leurs grandes priorités de recherche, et permet à des chercheurs plus isolés de mener à bien leurs travaux.

Plus d'une centaine de contrats passés avec divers partenaires ont permis de faire progresser les connaissances. 
Par exemple, d'importants travaux ont été menés sur la propagation des ondes radio dans la neige et ils ont largement contribué à la mise au point d'un «ARVA » français. De nombreuses études ont aussi été réalisées par le CENG (LASP) sur le déclenchement artificiel des avalanches (explosifs, bang supersonique...). L'ANENA a ainsi poussé, soutenu de toutes ses forces la mise au point de l'avalancheur, lanceur pneumatique d'explosifs, permettant le déclenchement des avalanches à distance.

Actuellement les domaines étudiés sont le transport de la neige par le vent et la répartition de la neige dans les zones de départ des avalanches, avec l'étude des fameuses plaques (à vent ou non), et la détection sismique des avalanches.

Force de proposition, de gestion, l'ANENA intervient également dans la mise en œuvre des résultats : soucieuse avant tout d'efficacité, elle se situe résolument à la jonction entre la recherche fondamentale et ses applications sur le terrain. Les symposiums précédemment cités s'insèrent également dans ce cadre.

\subsubsection{Un organisme de formation}

Depuis plus de vingt ans, l'ANENA forme les spécialistes artificiers en déclenchement préventif et artificiel des avalanches à l'aide d'explosifs.

Pisteurs-secouristes des stations de ski, agents de l'Equipement ou autres, ils sont environ 2000 (environ 80 par an) à avoir suivi le stage de formation et obtenu le Certificat de Préposé au Tir, option tir en montagne pour le déclenchement des avalanches.

L'Association dispense aussi la spécialisation « servant avalancheur " permettant aux artificiers de pouvoir utiliser ce «canon pneumatique ».

Sont également formés par l'ANENA les maîtres-chiens d'avalanches et leurs fidèles compagnons. Plus de 20 nouvelles équipes opérationnelles « sortent » ainsi chaque année d'un stage de 15 jours afin d'améliorer les secours en avalanches.

Enfin, l'Association organise chaque hiver un stage «fréquentation hivernale de la montagne " à l'intention des personnes dont la profession les amène à être fréquemment en montagne l'hiver (personnels des parcs nationaux, des services RTM...). Ces stages sont également ouverts au grand public.

\subsubsection{Un centre d'information}

L'information se situe à plusieurs niveaux. S'adressant à des lecteurs souvent professionnels et en tout cas très concernés, la revue « Neige et Avalanches » trimestrielle est d'un bon niveau scientifique et technique. De plus, le bulletin bibliographique qui y est publié permet à chacun de se tenir informé des nouvelles publications dans le large domaine de la neige, des avalanches et de la sécurité en montagne hivernale. Mais l'information interne ne suffit pas. Il faut aussi toucher le public. Dans ce domaine, I'ANENA doit adapter sa politique à ses moyens, qui restent limités. Les statistiques d'accidents permettent de définir des usagers « à risques » sur lesquels doivent porter en priorité, les actions d'information et de sensibilisation ; la tâche n'est pas aisée : si les randonneurs, «skieurs de printemps ", étaient relativement faciles à toucher par l'intermédiaire des clubs, il y a quelques années, aujourd'hui, la diversification des pratiques, leur étalement dans l'espace et dans le temps et surtout leur caractère de plus en plus individualiste, rendent l'information plus difficile. Le dépliant «ski et sécurité » (épuisé) tiré à plusieurs dizaines de milliers d'exemplaires, la campagne «ski et forêts " (sensibilisation du grand public sur l'impact du ski horspistes sur les forêts) et l'édition du livre "ski et sécurité " (épuisé) de François Valla, montrent cependant qu'il est possible de lancer des opérations efficaces. C'est certainement en partie grâce à ces actions que le nombre de victimes d'avalanches n'a guère augmenté au cours des dernières années, alors que le nombre de pratiquants (surtout en hors-pistes) a connu dans le même temps un fort accroissement.

\subsection{Le rayonnement international de l'ANENA}

L'ANENA a été rapidement appréciée dans les milieux montagnards spécialisés du monde entier et jouit maintenant d'une très bonne image de marque à l'étranger. $\mathrm{Cu}$ rieusement, sa formule souple, celle de l'Association, considérée à l'origine chez nous comme un pis-aller, voire un handicap, a même fait des envieux chez certains de nos interlocuteurs étrangers, qui ressentent parfois avec quelque peine le poids des structures plus directives.

Elle a même servi d'exemple pour la création d'une association analogue en Italie (AINEVA), et le Chili travaille depuis quelques années sur un projet semblable.

En plus de sa participation très active aux organismes internationaux précités, des symposiums, internationaux eux aussi, organisés par l'ANENA, l'Association est présente à l'étranger lors de grands congrès sur la neige et les avalanches, comme à l'International Snow Science Workshop (ISSW, colloque bisannuel nord américain sur la neige et les avalanches), ou le symposium de Manali en Inde, grâce à de nombreuses communications. Cette représentation à l'étranger permet de valoriser le savoir et le savoir-faire français. Certains ont ainsi vu dans l'ANENA une «équipe de France des avalanches ", image qui n'a rien d'excessif.

\section{$\mathrm{V}$ CONCLUSIONS}

Le fonctionnement de l'ANENA est donc à la fois complexe (de nombreux organismes y étant associés, se pose parfois le problème du " qui fait quoi ? ») et simple puisque basé sur la recherche d'une synergie. L'ANENA peut s'effacer derrière un partenaire ou donner l'impression de «tirer la couverture " à elle ; elle peut centraliser puis déléguer. C'est toute la difficulté d'une association nationale qui ne doit oublier aucun de ses partenaires et s'intéresser à tous les massifs, tout en trouvant un large consensus pour poursuivre sa route. Mais, en matière d'avalanches, tout n'est-il pas une question d'équilibre ! 